\title{
Levantamento da Fauna de Ichneumonidae (Hymenoptera) em Cultivo de Coqueiro Anão Verde Associado à Plantas Invasoras
}

\author{
Emerson Fraga Comério ${ }^{1}$, Helena Carolina Onody ${ }^{\bowtie}$ \& Vera Lúcia Rodrigues Machado Benassi ${ }^{3}$
}

\begin{abstract}
1. Universidade Estadual Paulista "Júlio de Mesquita Filho", Faculdade de Ciências Agrárias e Veterinárias de Jaboticabal, e-mail: emersoncomerio@hotmail.com. 2. Museu de Zoologia da USP, e-mail: helenaonody@yahoo.com.br (Autor para correspondência ${ }^{\bowtie}$ ). 3. Instituto Capixaba de Pesquisa, Assistência Técnica e Extensão Rural (Incaper), e-mail: vbenassi@incaper.es.gov.br
\end{abstract}

\section{EntomoBrasilis 5 (2): 109-114 (2012)}

Resumo. Este trabalho teve por objetivo realizar um levantamento da fauna de Ichneumonidae (Hymenoptera) em cultivo de coqueiro anão verde e avaliar a influência de plantas invasoras sobre esta fauna. As amostragens foram realizadas semanalmente durante o período de março/2008 a fevereiro/2009, utilizando armadilhas Möericke em uma área mantida roçada e outra com a presença de plantas invasoras. Foram obtidos 569 exemplares pertencentes a 11 subfamílias. Cryptinae representou mais de 50\% do material coletado nos dois ambientes e, dentre as subfamílias ophioniformes, nove gêneros foram identificados, sendo Anomalon e Dusona os mais abundantes. A área com plantas invasoras apresentou maior número de subfamílias e abundância de indivíduos coletados em relação à área mantida roçada. Entretanto, diferenças significativas na abundância foram constadas somente para Cryptinae e Ichneumoninae.

Palavras-Chave: Cocos nucifera; Controle biológico conservativo; Parasitoides.

\section{Survey of the Ichneumonidae (Hymenoptera) Fauna in Coconut Palm Crop Associated with Invasive Plants}

Abstract. This study aimed to survey the Ichneumonidae (Hymenoptera) fauna in coconut palm crop and analyze the influence of invasive plants on this fauna. From February 2008 to March 2009 weekly samples were performed using Möericke traps in areas with and without invasive plants. A total of 569 specimens were identified in 11 subfamilies. Cryptinae corresponded to more than $50 \%$ of the total ichneumonids collected in the two studied areas and, among the ophioniformes subfamilies, 9 genera were identified, being Dusona and Anomalon the most abundant. The area with invasive plants presented a higher number of subfamilies and abundance of specimens. However, significant differences in abundance were observed only to Cryptinae and Ichneumoninae.

Keywords: Cocos nucifera; Conservative biological control; Parasitoids.

$\mathbf{O}$ coqueiro, Cocos nucifera L. (Arecaceae), é uma planta tipicamente tropical originada do Sudeste Asiático e encontra-se atualmente disseminada em diversas regiões do globo. Considerado por muitos como "árvore da vida", constitui uma das principais culturas perenes capaz de gerar um sistema autossustentável de exploração (ARAGão et al. 2002; BENASSI 2006).

O estado do Espírito Santo é um dos maiores produtores da variedade anã verde. De uma produção estimada em 152,7 milhões de frutos destinados ao consumo de água, o estado é responsável pela comercialização de mais de sete mil toneladas por ano (AgRIANUAL 2008; INCAPER 2008).

O manejo fitossanitário dos cultivos de coco é de extrema importância. Segundo estudos de Ferreira et al. (1997), as pragas em coqueirais representam um grave problema e causam importantes danos às diferentes partes e estágios de desenvolvimento da planta. A ação nociva das pragas pode ser observada desde a implantação da cultura, ocasionando elevado número de replantios e atraso no desenvolvimento vegetativo. $\mathrm{O}$ problema agrava-se à medida que a planta entra em produção e atinge a fase adulta, pois as pragas também podem atacar as inflorescências, flores e frutos em diferentes estágios de maturação (FERREIRA et al. 1997; FERREIRA 2002).
De maneira geral, o controle de pragas agrícolas tem sido feito principalmente através do manejo convencional, com a utilização de produtos químicos (Matson et al. 1997). Entretanto, o uso abusivo desses insumos é altamente prejudicial provocando intoxicação de animais e seres humanos, degradação do solo, acúmulo de resíduos químicos nos produtos, ressurgimento de pragas e patógenos, desorganização da dinâmica populacional, perda da biodiversidade e resistência de pragas (AlTIERI 1995, 1999; FAdini et al. 2001; BarBosa et al. 2003).

Recentemente, estudos têm sugerido a conciliação da exploração de ambientes por atividades agrícolas com a conservação da biodiversidade, promovendo uma série de benefícios, como maior resiliência e a obtenção de sustentabilidade ao longo do tempo (Paolleti et al. 1992; Altieri 1995).

Nos agroecossistemas, as plantas invasoras constituem importante componente da diversidade florística. Em áreas de manejo convencional, estas plantas são por algumas vezes consideradas prejudiciais por exercerem estresse biótico direto sobre as culturas, competindo por luz, umidade e nutrientes. Além disso, algumas espécies podem servir como hospedeiras de algumas pragas agrícolas (ALTieri et al. 2003).

Por outro lado, no contexto do manejo ecológico as plantas invasoras podem ser benéficas, pois auxiliam na diminuição 
de perdas de solo por erosão e influenciam positivamente os inimigos naturais, fornecendo recursos para a sua sobrevivência e reprodução e, consequentemente, impondo maior mortalidade às pragas (Garcia 2001; Altieri et al. 2003; Aguiar-Menezes 2004, 2006; Pfiffner \& Wyss 2004). Por isso, o sucesso deste tipo de manejo é altamente dependente do estudo das plantas invasoras em cultivos, bem como de sua fauna de inimigos naturais associada.

Dentre os inimigos naturais, os parasitoides constituem importantes agentes de controle de pragas agrícolas e atuam como espécies-chave nos agroecossistemas (LASALLE 1993). Muitas espécies de parasitoides têm sido utilizadas em programas de controle biológico devido à eficiência com que localizam e exploram seus hospedeiros.

Considerando o potencial benéfico da presença de parasitoides e plantas invasoras em agroecossistemas no controle de pragas, o presente trabalho teve por objetivo realizar levantamento da fauna de Ichneumonidae associada ao cultivo de coqueiro anão verde e avaliar a influência da presença de plantas invasoras sobre ela.

\section{MATERIAL E MÉTODOS}

O trabalho foi realizado em uma lavoura de Cocos nucifera L. (Arecaceae), variedade anã verde na Fazenda Experimental do Instituto Capixaba de Pesquisa, Assistência Técnica e Extensão Rural (19² 25'06,959” S 40 04' 30,026” W) em Linhares, ES, Brasil. A lavoura de cinco hectares possui plantas com idade de 9 anos, dispostas em arranjo triangular e espaçadas 7,5 m entre si. A classificação climática da região segundo Köeppen é Aw, tratando-se de clima tropical úmido com inverno seco é tropical quente e seco (Nóbrega et al. 2012).

As amostragens foram realizadas semanalmente no período de março/2008 a fevereiro/2009 em duas áreas de $15 \mathrm{~m}$ x $30 \mathrm{~m}$ cada e distantes $37,5 \mathrm{~m}$. Uma área foi mantida roçada e outra com plantas invasoras e, em cada uma, a cada $22,5 \mathrm{~m}$ foram instaladas seis armadilhas Möericke (39 cm comprimento x 29 $\mathrm{cm}$ largura x $6 \mathrm{~cm}$ profundidade), de cor amarela, colocadas ao nível do solo, sob a copa das plantas, preenchidas com água $(1,5$ L), formaldeído $37 \%$ (15 mL) e gotas e detergente neutro.

O material coletado foi transportado para o laboratório de Controle Biológico do INCAPER onde os Ichneumonidae foram triados e conservados em frascos plásticos contendo álcool $70 \%$. Para a identificação das subfamílias foi utilizado o trabalho de HANSON \& GAULd (2006). Somente as subfamílias ophioniformes foram identificadas até gênero, uma vez que este grupo possui grande quantidade de espécies parasitoides de importantes pragas agrícolas.

As plantas invasoras foram observadas quinzenalmente e identificadas com auxílio do Manual de Identificação e Controle de Plantas Daninhas (LoRENZI 2008).

A análise de correlação de Spearman foi utilizada para avaliar a influência de variáveis climáticas na abundância de ichneumonídeos nas duas áreas estudadas. Os dados de temperatura, umidade e precipitação média dos meses em que as armadilhas ficaram expostas foram fornecidos pelo Centro de Meteorologia e Recursos Hídricos do INCAPER. Também foram realizados testes não paramétricos de Kruskall-Wallis para comparar as diferenças na abundância das subfamílias entre as duas áreas. Ambas as análises foram feitas utilizando o programa estatístico BioEstat 5.0 (Ayres et al. 2007).

Para a análise faunística dos gêneros ophioniformes identificados foram calculados os índices de ocorrência e dominância segundo Palma (1975) apud Abreu \& Nogueira (1989). A ocorrência dos gêneros foi agrupada em três classes: acidental (o,o a 25,0 $\%$ ), acessória (25,0 a 50,0 \%) e constante (50,0 a 100,0 \%) e a dominância, também, em três classes: acidental (o,o a 2,5 \%), acessória ( 2,5 a 5,0 \%) e dominante (5,0 a 100,0 \%). A combinação destes dois índices permitiu classificar os gêneros em: comum, quando constante e dominante; intermediário, quando constante e acessório, constante e acidental, acessório e acidental, acessório e dominante, acessório e acessório; e raro, quando acidental e acidental.

\section{RESULTADOS E DISCUSSÃO}

Foram coletados 569 ichneumonídeos identificados em 11 subfamílias (Tabela 1). Dentre estas, oito pertencem aos ophioniformes (Anomaloninae, Campopleginae, Cremastinae, Mesochorinae, Metopiinae, Nesomesochorinae, Ophioninae e Tryphoninae), duas aos ichneumoniformes (Cryptinae e Ichneumoninae) e uma aos pimpliformes (Pimplinae), segundo agrupamento proposto por Quicke et al. (2009).

Todas as onze subfamílias foram identificadas na área com plantas invasoras, onde a abundância de indivíduos foi três vezes maior; já na área mantida roçada, Mesochorinae, Nesomesochorinae e Ophioninae não foram coletadas. O teste de Kruskal-Wallis apontoudiferençassignificativasnaabundânciaentreosambientes para Cryptinae $(\mathrm{p}=0,004)$ e Ichneumoninae $(\mathrm{p}=0,002)$, mas não para Anomaloninae $(\mathrm{p}=0,416)$; Campopleginae $(\mathrm{p}=0,334)$ Pimplinae $(\mathrm{p}=0,58)$, Cremastinae $(\mathrm{p}=1)$; Mesochorinae $(\mathrm{p}=0$, 148); Metopiinae ( $\mathrm{p}=0,135)$; Nesomesochorinae $(\mathrm{p}=0,713)$; Ophioninae $(\mathrm{p}=\mathrm{o}, 344)$ e Tryphoninae $(\mathrm{p}=\mathrm{o}, 139)$.

A flutuação populacional dos ichneumonídeos ao longo do estudo mostrou que, na área com plantas invasoras os meses de setembro/2008 e fevereiro/2009 foram os que apresentaram maior captura de indivíduos, enquanto que maio e julho/2008 foram os meses de menor captura (Figura 1). Na área mantida roçada, o maior número de indivíduos foi coletado nos meses de agosto/2008 e fevereiro/2009 enquanto nos meses de maio, dezembro/2008 e janeiro/ 2009 não houve indivíduos coletados.

Não foi observada correlação significativa entre abundância de ichneumonídeos e variáveis climáticas na área com plantas invasoras [temperatura média ( $\mathrm{rs}=0.2523, \mathrm{p}=0.4289$ ), umidade relativa ( $\mathrm{rs}=-0,3209, \mathrm{p}=0.3092)$ e precipitação $(\mathrm{rs}=0.0141$, $\mathrm{p}=0.9652)]$ e, na área mantida roçada, somente para umidade relativa [temperatura média $(\mathrm{rs}=-0.2058, \mathrm{p}=0.5211)$, umidade relativa $(\mathrm{rs}=-0,6554, \mathrm{p}=0.0207)$ e precipitação $(\mathrm{rs}=-0.4496, \mathrm{p}=$ $0.1425)]$.

É preciso salientar, no entanto, que os resultados obtidos neste trabalho podem ter sofrido influência da metodologia empregada nas coletas. Estudos recentes têm demonstrado que Möericke e Malaise, armadilhas mais comumente utilizadas em amostragem de parasitoides, podem apresentar resultados diferentes em relação aos táxons e sexo de ichneumonídeos amostrados (MAZón \& Bordera 2008; Aguiar \& SANTOS 2010), reforçando, portanto, a necessidade da combinação dos métodos de coleta para a determinação e monitoramento da fauna.

A subfamília Cryptinae foi a mais abundante nos dois locais estudados e representou mais de 50\% do material coletado. Esta é a maior subfamília de Ichneumonidae, com grande número de espécies na região neotropical, e que possuem biologia e associações hospedeiras muito amplas (GAUlD 2006; PALACiO \& WAHL 2006). Ichneumoninae, cujas espécies se desenvolvem principalmente como endoparasitoides de Lepidoptera, foi relativamente abundante constituindo a segunda subfamília mais amostrada (17,5\%). Já Pimplinae, uma das subfamílias mais diversas biologicamente (GAULD 1991) apresentou baixa abundância. Porém, a ocorrência desta subfamília pode ser de grande benefício para os cultivos de coco já que existe registro de Pedinopelte gravenstii Guérin-Ménéville, parasitando a praga do coqueiro Automeris cinctistriga Felder (FERREIRA et al. 1997). 
Tabela 1. Ichneumonídeos coletados em cultura de coqueiro anão verde, no período de março/2008 a fevereiro/2009 e classificação de acordo com os índices de ocorrência e dominância (CP: área com plantas invasoras; SP: área mantida roçada; T: total de ichneumonídeos coletados; c; comum; i: intermediário; r: raro).

\section{Subfamília}

CP

SP

$\mathbf{T}$

OPHIONIFORMES

\section{Anomaloninae}

Anomalon Panzer, 1804

62

(c)

19

(c)

81

\section{Campopleginae}

Dusona Cameron, 1900

23

(c)

(c)

Microcharops Roman, 1910

02

(i)

10

(i)

07

\section{Cremastinae}

Eiphosoma Cresson, 1865

03

(i)

03

\section{Mesochorinae}

Mesochorus Gravenhorst, 1829

03

(r)

o

( $r)$

\section{Metopiinae}

Colpotrochia Holmgren, 1855

08

(i)

03

\section{Nesomesochorinae}

Nonnus Cresson, 1874

o

( $\mathrm{r}$ )

\section{Ophioninae}

Enicospilus Stephens, 1835

06

(i)

o

(r)

Tryphoninae

Netelia Gray, 1860

04

(i)

01

(r)

\section{ICHNEUMONIFORMES}

\begin{tabular}{|lcccc} 
Cryptinae & 221 & 81 & 302 \\
\hline Ichneumoninae & 82 & 18 & 100
\end{tabular}

PIMPLIFORMES

\section{Pimplinae}

Total 08

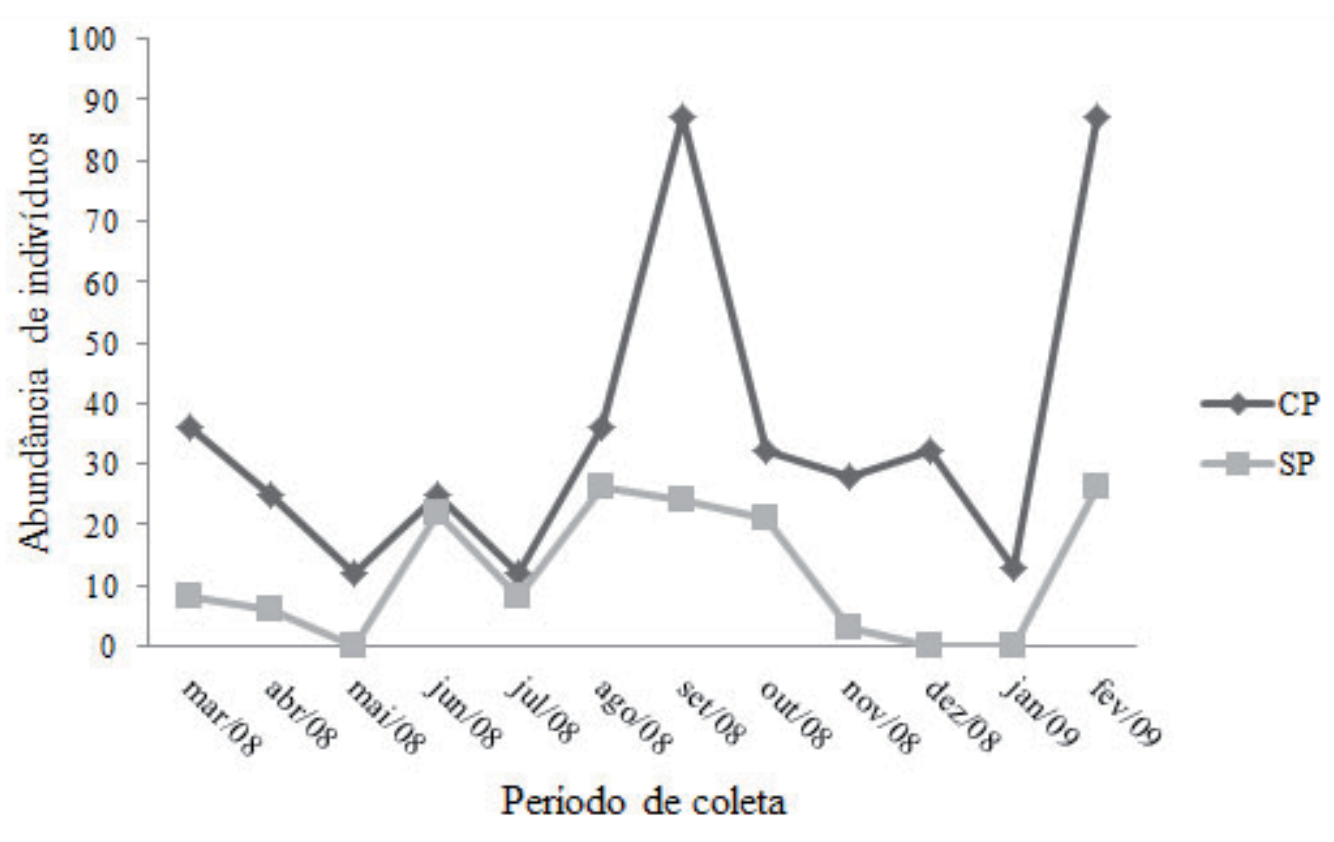

Figura 1. Flutuação populacional de ichneumonídeos capturados com armadilhas Möericke em áreas de cultivos de coqueiro anão verde em Linhares, ES, de março de 2008 a fevereiro de 2009. (CP: área com plantas invasoras; SP: área mantida roçada). 
Dentre os ophioniformes, Anomaloninae e Campopleginae foram os mais abundantes. Os Anomaloninae frequentemente se desenvolvem como endoparasitoides cenobiontes de larvas de Lepidoptera, porém todos os exemplares coletados foram identificados como Anomalon, gênero que também possui espécies que atacam larvas Coleoptera. Esse fato pode ter importância para o manejo ecológico de pragas, pois o besouro Taphrocerus cocois Bondar (Buprestidae), também conhecido como minador-do-folíolo, é considerado praga da cultura (FERREIRA et al. 1997).

Apesar da sua abundância em agroecossistemas (SANDONATo et al. 2010) somente dois gêneros de Campopleginae foram coletados neste estudo: Microcharops e Dusona. O parasitismo por Microcharops tem sido registrado para noctuídeos considerados pragas agrícolas, como Alabama argillacea (Hübner), Rachiplusia nu (Guenée), Spodoptera frugiperda (Smith), Trichoplusia ni (Hübner) e Anticarsia gemmatalis Hübner; o gênero Dusona possui uma grande diversidade de hospedeiros, a maioria noctuídeos, notodontídeos e geometrídeos (Yu et al. 2005).

As demais subfamílias representaram menos de 10\% do material coletado e tiveram somente um gênero identificado cada: Eiphosoma Cresson (Cremastinae), Netelia (Tryphoninae), Colpotrochia (Metopiinae), Nonnus (Nesomesochorinae), Enicospilus (Ophioninae) e Mesochorus (Mesochorinae).

Dentre estes gêneros, algumas espécies já foram registradas parasitando importantes pragas agrícolas: espécies de Eiphosoma parasitando $S$. frugiperda e Hellula phidilealis (Walker); espécies de Netelia parasitando Agrotis ipsilon (Hufnagel), S. frugiperda e Helicoverpa zea (Boddie), e espécies de Enicospilus parasitando Manduca sexta (Linnaeus), Diatraea saccharalis (Fabricius), $T$. ni, A. ipsilon, S. frugiperda, H. zea e Automeris (Yu et al. 2005). Automeris é um gênero saturnídeo que possui uma espécie, $A$. Automeris cinctistriga Felder, que ataca o coqueiro anão verde (FERREIRA et al. 1997).
Analisando a frequência de ocorrência e dominância dos gêneros ophioniformes capturados verificou-se que somente Anomalon e Dusona foram constantes nos dois ambientes (Tabela 1). A maioria dos gêneros foi classificada como intermediária, exceto Mesochorus que foi rara em ambos locais estudados.

As plantas invasoras que ocorreram durante o período de levantamento foram identificadas em oito famílias e quinze gêneros, sendo a identificação das espécies possível somente para treze deles (Tabela 2). A família Asteraceae apresentou maior número de espécies floridas durante quase todo o ano, o que pode favorecer a fauna de inimigos naturais no local. De acordo com BRombal (2001), as asteráceas e outras dicotiledôneas são comuns em agroecossistemas, e se caracterizam por terem uma flora extensa ao longo do ano, sendo que suas flores possuem características que permitem fácil acesso ao pólen por grande número de parasitoides.

Altieri et al. (2003) ressaltam também a importância de outras plantas como Cassia, Euphorbia e Borreria identificadas neste estudo, e sua utilização em programas de manejo ecológico de pragas em algumas culturas.

As plantas invasoras floríferas em culturas agrícolas podem ser importantes para o fornecimento de alimento suplementar às fêmeas de Ichneumonidae, promovendo o aumento da fecundidade, longevidade e, consequentemente, de sua abundância nos campos cultivados. VAM ENDEM (1965) relatou que algumas fêmeas devem alimentar-se de néctar para maturar os ovos. Outros estudos verificaram que a fecundidade e a longevidade de algumas espécies aumentam significativamente com a presença de plantas invasoras (Syme 1975; Johanowicz \& Mitchell 2000).

Pesquisas nos últimos anos têm demonstrado que a presença de inimigos naturais reduz surtos de certos tipos de pragas agrícolas

Tabela 2. Plantas invasoras registradas em cultura de coqueiro anão verde em Linhares, ES, no período de março/2008 a fevereiro/2009.

\begin{tabular}{|c|c|c|}
\hline Família / espécie & Nome vulgar & Ocorrência \\
\hline \multicolumn{3}{|l|}{ Asteraceae } \\
\hline Ageratum conyzoides L. & picão-roxo & mar a jul - set,out \\
\hline Bidens pilosa $\mathrm{L}$. & picão-preto & mar a jun - set, out, dez \\
\hline Emilia sanchifolia (L.) DC. & falsa-serralha & mar a ago \\
\hline Sonchus oleraceus L & serralha & mar a ago \\
\hline \multicolumn{3}{|l|}{ Amaranthaceae } \\
\hline Alternanthera tenella Colla & apaga-fogo & maio a out \\
\hline \multicolumn{3}{|l|}{ Commelinacea } \\
\hline Commelina benghalensis L. & trapoeraba & mar, jun, jul, set, out \\
\hline \multicolumn{3}{|l|}{ Convolvulaceae } \\
\hline Ipomoea sp. & corda-de-viola & maio \\
\hline \multicolumn{3}{|l|}{ Euphorbiaceae } \\
\hline Euphorbia hirta L. & erva-de-santa-luzia & out \\
\hline \multicolumn{3}{|l|}{ Fabaceae } \\
\hline Cassia hirsuta L. & fedegoso-peludo & mar, jun, jul, set, out \\
\hline Desmodium barbatum (L.) & barbadinho & mar, jul \\
\hline Indigofera hirsuta L. & amores-do-campo & Jun \\
\hline \multicolumn{3}{|l|}{ Malvaceae } \\
\hline Sida sp. & guanxuma & fev, abr, jul, dez \\
\hline \multicolumn{3}{|l|}{ Rubiaceae } \\
\hline Borreria verticillata (L.) & vassourinha & maio \\
\hline Lantana camara L. & erva-chumbinho & jun \\
\hline Stachytarphetta cayenensis (Rich.) M. & gervão & fev a abr, - jun a dez \\
\hline
\end{tabular}


devido à maior mortalidade imposta por estes agentes. Neste trabalho, o levantamento da ichneumofauna associada a cultivo de coco identificou a ocorrência de grupos potencialmente importantes no controle de pragas agrícolas, apesar de não ter sido conclusivo em relação ao impacto de plantas invasoras sobre esta fauna.

\section{AGRADECIMENTOS}

Ao pesquisador Márcio Adonis Miranda Rocha do INCAPER de Vitória, ES pela confirmação da identificação das plantas invasoras, ao CNPq pela bolsa de pós-doutoramento concedida e aos dois revisores anônimos que contribuíram com suas sugestões para a melhoria do manuscrito.

\section{REFERÊNCIAS}

Abreu, P.C.O.V. \& C.R. Nogueira, 1989. Spatial distribution of Siphonophora species at Rio de Janeiro Coast, Brazil. Ciência e Cultura, 41: 897-902.

Agrianual, 2008. Coco-da-baía. p. 286-292. In: Anuário da Agricultura Brasileira. São Paulo, FNP, Consultoria e Agroinformativos, 516p.

Aguiar, A.P.\& B.F. Santos, 2010. Discovery of potent, unsuspected sampling disparities for Malaise and Möricke traps, as shown for Neotropical Cryptini (Hymenoptera, Ichneumonidae). Journal of Insect Conservation, 14: 199-206.

Aguiar-Menezes, E.L., 2004. Diversidade Vegetal: uma estratégia para o manejo de pragas em sistemas sustentáveis de produção agrícola. Embrapa Agrobiologia, Seropédica, (Documentos, 177), $68 \mathrm{p}$.

Aguiar-Menezes, E.L., 2006. Controle Biológico: na busca pela sustentabilidade da agricultura brasileira. Campo \& Negócios, 4: 66-77.

Altieri, M.A., 1995. Agroecology: the science of sustainable agriculture. Colorado, Westview Press, Bolder, 435p.

Altieri, M.A., 1999. The ecological role of biodiversity in agroecosystems. Agriculture, Ecosystems and Environment, 74: 19-31.

Altieri, M.A., E.N. Silva \& C.I. Nicholls, 2003. O Papel da Biodiversidade no Manejo Integrado de Pragas. Ribeirão Preto, Holos, 226 p.

Aragão, W.M., F.E. Ribeiro, E.A. Tupinambá \& E.R. de Siqueira, 2002. Variedades e híbridos do coqueiro. p. 26-34. In: Aragão, W.M. (Ed.). Coco pós-colheita. Brasília: EMBRAPA (Série Frutas do Brasil), 76p.

Ayres, M., M. Ayres Junior, D.L. Ayres \& A.A.S. Santos, 2007. BioEstat: aplicações estatísticas nas áreas das Ciências Biomédicas. Belém, Sociedade Civil Mamirauá, 324p.

Barbosa, F.R., R.G. Ferreira, L.H.P. Kill, E.A. Souza, W.A. Moreira, J.A. Alencar \& F.N.P. Haji, 2003. Nível de Dano, Plantas Invasoras Hospedeiras, Inimigos Naturais e Controle do Psilídeo da Goiabeira (Triozoida sp.) no Submédio São Francisco. Revista Brasileira de Fruticultura, 25: 425-428.

Benassi, A.C., 2006. Coco: Informe sobre a produção de coco. Disponível em: <www.todafruta.com.br $>$ Acesso em: 22 nov. 2009.

Brombal, J.C., 2001. Estudo da fauna de Artrópodes Associada a plantas Invasoras em Agroecossistemas Orgânicos e Convencionais. Dissertação (Mestrado em Ecologia) Universidade Estadual de Campinas. 141p.

Fadini, M.A.M., M.A. Regina, J.C. Fráguas \& J.N.C. Louzada, 2001. Efeito da Cobertura Vegetal do Solo sobre a Abundância e Diversidade de Inimigos Naturais de Pragas em Vinhedos. Revista Brasileira de Fruticultura, 23: 573-576.

Ferreira, J.M.S., 2002. Coco: fitossanidade. Embrapa Informação tecnológica, $136 \mathrm{p}$.

Ferreira, J.M.S., R.N. Warnick, \& L.A. Siqueira, 1997. A cultura do coqueiro no Brasil. Brasília, EMBRAPA, 306p.

Garcia, M.A., 2001. Ecologia aplicada a agroecossitemas como base para a sustentabilidade. Informe Agropecuário, 22: 3038.
Gauld I.D., 2006. Familia Ichneumonidae. p. 446-487. In: Hanson P. \& I.D. Gauld (eds) Hymenoptera de la Región Neotropical. Memoirs of the American Entomological Institute, Gainesville 77, 994p.

Gauld, I.D., 1991. The Ichneumonidae of Costa Rica, 1. Memoirs of the American Entomological Institute, 47: 589p.

Hanson, P.Y. \& I.D. Gauld, 2006. Hymenoptera de la Región Neotropical. Memories of the American Entomological Institute. Gainesville 77, 994p.

INCAPER, 2008. Produtividade de coco no Espírito Santo é duas vezes maior que a média nacional Disponível em: <http:// www.seag.es.gov.br/?p=2247> Acesso em: 06 mar. 2008.

Johanowicz, D. \& E.R. Mitchell, 2000. Effects of sweet alyssum flowers on the longevity of the parasitoid wasps Cotesia marginiventris (Hymenoptera: Braconidae) and Diadegma insulare (Hymenoptera: Ichneumonidae). Florida Entomologist, 83: 41-47.

LaSalle, J., 1993. Parasitic Hymenoptera, biological control and biodiversity, p. 197-215. In: Lasalle, J. \& I.D. Gauld (Eds.). Hymenoptera and biodiversity. Wallingford, $\mathrm{CAB}$ International, IOBC, 348p.

Lorenzi, H., 2008. Plantas Daninhas do Brasil. 4. ed. São Paulo: Instituto Plantarum, $672 \mathrm{p}$.

Matson, P.A., A.G. Parton, M.J. Power, \& M.L. Swift, 1997. Agricultural Intensification and Ecossystems Proprierties. Science 277: 504-508.

Mazón, M. \& S. Bordera, 2008. Effectiveness of two sampling methods used for collecting Ichneumonidae (Hymenoptera) in Cabañeros National Park (Spain). European Journal of Entomology 105: 879-888.

Nóbrega, N.E.F., J.G.F. da Silva, H.E.A. Ramos \& F.S. Pagung. 2012. Balanço hídrico climatológico e classificação climática de Thornthwaite e Köppen para o município de Linhares. ES. Disponível em http://hidrometeorologia.incaper.es.gov.br/ arquivos pdf/publicacoes/CONIRD/1567 linhares.pdf.> Acesso em: 06 mar. 2012.

Palacio, E.E. \& D.B. Wahl, 2006. Familia Ichneumonidae. p. 293329. In: Fernández, F. \& M.J. Sharkey (Eds). Introduccion a los Hymenoptera de la Region Neotropical. Sociedad Colombiana de Entomologia y Universidad Nacional de Colombia, xxx + 894p.

Paoletti, M.G., B.R. Pimentel \& D. Stiner, 1992. Agroecosystem Biodiversity: Matching Production and Conservation Biology. Agriculture, Ecosystems e Environment, 40, 3-23.

Pfiffner, L. \& E. Wyss, 2004. Use of sown wildflower strips to enhance natural enemies of agricultural pests, p. 167-188. In: Gurr G.M., S.D. Wratten \& M.A. Altieri (Eds). Ecological Engineering for Pest Management: Advances in Habitat Manipulation for Arthropods. CSIRO Publishing, 256p.

Quicke, D.L.J., N.M.L. Laurenne, M.G. Fitton \& G.R. Broad, 2009 A thousand and one wasps: a 28S rDNA and morphological phylogeny of the Ichneumonidae (Insecta: Hymenoptera) with an investigation into alignment parameter space and elision. Journal of Natural History, 43: 1305-1421.

Sandonato, D.L., H.C. Onody \& A.M. Penteado-Dias, 2010. Fauna de Campopleginae (Hymenoptera, Ichneumonidae) em hortas orgânicas em Araraquara e São Carlos, SP, Brasil. Biota Neotrópica, 10: 117-121.

Syme, P.D., 1975. The effects of flowers on the longevity and fecundity of two native parasites of the European pine shoot moth in Ontario. Environmental Entomology, 4: 337-346.

Van Enden, H.F., 1965. The role of uncultivated land in the biology of crop pests and beneficial insects. Scientific Horticulture, 17: $121-136$.

Yu, D.S., K. Van Achterberg \& K. Horstmann, 2005. World Ichneumonoidea 4. Taxonomy, biology, morphology and distribution. Taxapad 2005 (Scientific names for information management). Interactive electronic catalogue on DVD/CDROM. Taxapad, Vancouver. 
Recebido em:05/10/2011

Aceito em: 30/03/2012

Como citar este artigo:

Comério, E.F., H.C. Onody \& V.L.M. Benassi, 2012. Levantamento da Fauna de Ichneumonidae (Hymenoptera) em Cultivo de Coqueiro Anão

Verde Associado à Plantas Invasoras. EntomoBrasilis, 5(2): 109-114.

Acessível em: http://www.periodico.ebras.bio.br/ojs/index.php/ebras/article/view/196
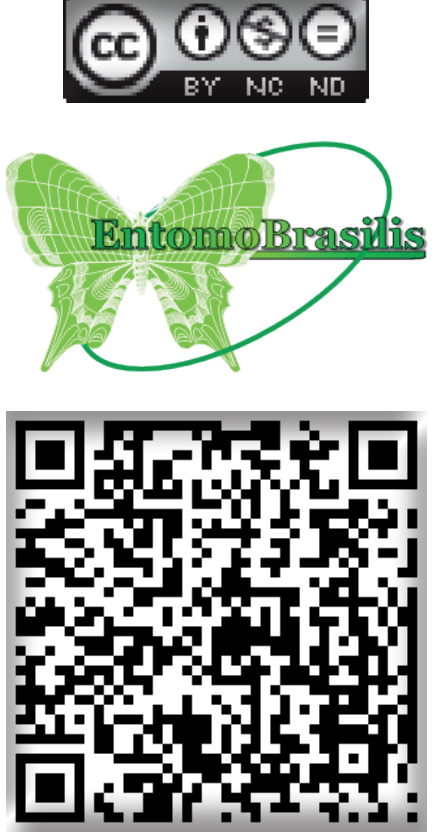